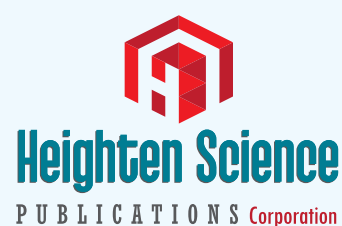

ISSN

2640-2874

\title{
Histological clonal change - A feature for dysplasia diagnosis
}

\author{
Lewei Zhang $^{1-3 *}$, Tarinee Lubpairee ${ }^{1,3}$, Denise M Laronde ${ }^{1,3,4}$, \\ Martial Guillaud ${ }^{3,4}$, Calum E MacAulay ${ }^{3,4}$ and Miriam P Rosin ${ }^{3-5}$ \\ 'Department of Oral Biological and Medical Sciences, Faculty of Dentistry, the University of \\ British Columbia (BC), Vancouver, Canada \\ BC Oral Biopsy Service, Vancouver General Hospital, Vancouver, Canada \\ ${ }^{3}$ BC Oral Cancer Prevention Program, BC Cancer Agency, Vancouver, Canada \\ ${ }^{4} \mathrm{BC}$ Cancer Research Centre, Vancouver, Canada \\ ${ }^{5}$ School of Biomedical Physiology and Kinesiology, Simon Fraser University, Burnaby, Canada
}

\begin{abstract}
*Address for Correspondence: Lewei Zhang, Department of Oral Biological and Medical Sciences, Faculty of Dentistry, University of British Columbia, BC Oral Biopsy Service, Vancouver General Hospital, BC Oral Cancer Prevention Program, BC Cancer Agency, 2199 Wesbrook Mall, Vancouver BC, Canada, V6T 1Z3, Tel: +1-604-875-4318 / 778-387-3885; Email: leweizhang888@gmail.com
\end{abstract}

Submitted: 23 September 2017

Approved: 27 August 2018

Published: 28 August 2018

Copyright: $\odot 2018$ Zhang L, et al. This is an open access article distributed under the Creative Commons Attribution License, which permits unrestricted use, distribution, and reproduction in any medium, provided the original work is properly cited

Keywords: Dysplasia diagnosis; Longitudinal studies; Loss of heterozygosity; Toluidine blue

Check for updates

\section{Abstract}

Aims: Histological diagnostic criteria are used for the assessment of the degree of dysplasia and hence the risk of cancer progression for premalignant lesions. Clonal changes in the form of hyperorthokeratosis and hyperchromasia that are sharply demarcated from adjacent areas are not currently part of the criterion for dysplasia diagnosis. The objective of this study was to determine whether such clonal change should be regarded as a diagnostic feature for dysplasia. The following histological conditions were used to define such change: (1) hyperorthokeratosis; (2) hyperchromatism but no other features of dysplasia; (3) sharp margin demarcation from adjacent area by both the hyperorthokeratosis and hyperchromasia (clonal change), and (4) no prominent rete ridges, marked acanthosis or heavy inflammation. Lesions fitting these criteria were termed orthokeratotic lesions with no dysplasia.

Methods: Patients from a population-based longitudinal study with more than 10 years of follow up were analyzed. Of the 214 patients with primary oral premalignant lesions, 194 had mild or moderate dysplasia (dysplasia group) and 20 fit the criteria for orthokeratotic lesions without dysplasia (orthokeratotic with no dysplasia group). The two groups were compared for their cancer risks using clinical (site and toluidine blue), histological (nuclear phenotype score), and molecular criteria (loss of heterozygosity) and by outcome (progression).

Results and conclusions: The lesions from orthokeratotic with no dysplasia group showed a similar cancer risk (clinical, histological and molecular risk) and time to progression as the dysplastic lesions. We recommend that the clonal change should be included as a criterion for dysplasia diagnosis.

\section{Introduction}

Squamous cell carcinoma (SCC) is the most common human cancer and also the most common oral cancer. SCCs often develop from premalignant lesions and the prediction of risk of malignant transformation for such lesions is critical to intervening in the course of the disease. The current gold standard for assessing such risk is by histological assessment of the presence and degree of dysplasia. The World Health Organization (WHO) and other authorities have established well-accepted diagnostic criteria for dysplasia [1,2]. However, there is still debate about other features of significance that should be included in the criteria. It is well known that non-dysplastic oral lesions, particularly those with hyperorthokeratosis, can also progress to cancer.

In one paper Woo et al. [3],suggested that "lesions that were keratotic but did not have significant evidence of inflammation, vascular ectasia, or fibrosis" be called "keratosis of unknown significance (KUS)" to alert clinicians to the possible premalignant nature 
of these lesions. Among cases that were presented in Woo's paper[3], there were hyperorthokeratotic lesions with a sharply demarcated histological margin that would not be called dysplastic by most if not all pathologists according to WHO dysplasia criteria. In our paper, we focus on this subgroup of KUS, i.e. hyperorthokeratotic lesions with no dysplasia (OKND) but with sharp histological demarcation. If such a lesion is located on the ventral tongue or floor of mouth, some would call it sublingual keratosis, an entity well recognized for its significant malignant potential [4].

The problem with calling these lesions names other than dysplasia is that clinicians may not understand the significance of such terms. In the BC Oral Biopsy Service, we routinely call OKND lesions dysplasia. There are two reasons for doing so: 1) the lesion is hyperchromatic, a feature well accepted for the diagnosis of dysplasia; and 2) the lesion is clonal, as shown by its sharp histological demarcation - clonal would suggest a neoplastic process (Figure 1). The clonal feature, however, has not been included in the WHO criteria for the diagnosis of dysplasia. Through the study results of this paper, we hope to persuade the pathology community to consider the possibility of inclusion of such clonal change as one of the criteria for the diagnosis of dysplasia.

The study of OPLs has been the focus of our research team for more than 2 decades, mainly with respect to the development of markers that would help in differentiating progressing from non-progressing mild/moderate dysplasia. The markers included clinical visual aids, such as TB staining [5,6], histological markers, such as nuclear phenotype score (NPS) [7,8], and molecular markers such as loss of heterozygosity (LOH) [9-12].

TB has long been used clinically to identify cancer and high-grade preinvasive lesions by staining these lesions blue. It was not, however, recommended for use with mild/moderate dysplasia because a high proportion of these lesions are negative for TB. The theory is that this dye stains nuclear acid that are present in abundance in the superficial epithelial layers of CIS and severe dysplasia but are most likely absent for mild/moderate dysplasia. In 2005, we reported that TB positive mild/moderate dysplasia had a 4-fold increase in cancer risk compared to histologically similar ТВ negative lesions [9]. It is hypothesized that the TB positive mild/moderate dysplasia may have increased inter-cellular permeability to allow the retention of the dye.

NPS is determined by semi-automatic computer image analysis of nuclear morphology. The analysis not only eliminates the subjectivity of dysplasia grading by pathologists but also could identify nuclear changes that differentiate progressing from non-progressing mild/moderate dysplasia that are too subtle for pathologists to determine and quantify. In 2008, we reported that mild/moderate dysplasia with high NPS scores had a 10-fold increase in cancer risk compared to histologically similar lesions with low NPS scores [7].

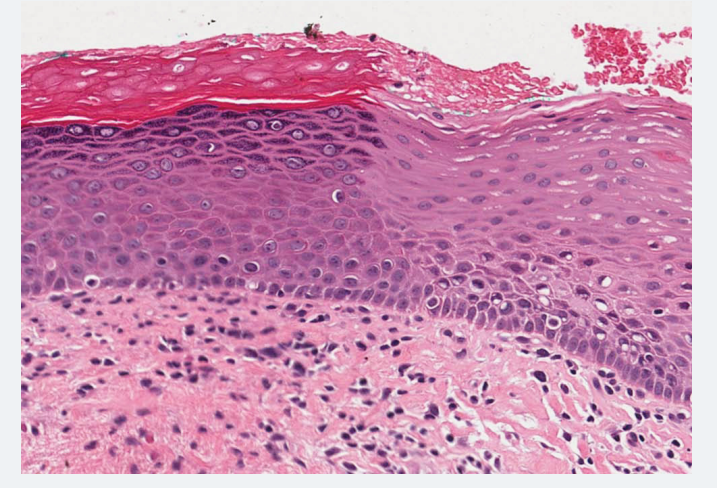

Figure 1: Photomicrograph of an orthokeratotic lesion without dysplasia (OKND) showing (1) hyperorthokeratosis (2) hyperchromatism but no other features of dysplasia; (3) sharp margin demarcation from adjacent epithelium by both the hyperorthokeratosis and by the hyperchromatism, and (4) lack of prominent rete ridges, marked acanthosis and heavy inflammation. Hematoxlin and eosin (HE staining). 
Microsatellite analysis of LOH identifies loss of chromosomal regions that contain putative tumor suppressor genes. Recently we published our ten-year follow up data on LOH as molecular markers for predicting cancer progression of OPLs [9]. In that paper, we not only validated the progression model we proposed in 2000 [12], but also refined the model.

The objective of this study was to determine if TB staining, NPS score, LOH pattern and progression rate of OKND lesions were similar to those of mild/moderate dysplasia, hence provide evidence in support of the inclusion of the clonal feature of such lesions in the criteria for the diagnosis of dysplasia.

\section{Materials and Methods}

\section{Study design}

Prospective cohort.

\section{Patient population}

The study involved patients from a previously published longitudinal study involving 296 patients with primary mild/moderate dysplasia [9] (approved by the University of British Columbia and BC Cancer Agency Research Ethics Board). This population-based study involved patients who prospectively enrolled in the ongoing Oral Cancer Prediction Longitudinal study in Vancouver (British Columbia, Canada) between January 1, 1997 and December 31, 2007. Accrual to this cohort was from community practices across British Columbia (population, 4.1 million in 2011). Patients were identified primarily through a centralised pathology service, the BC Oral Biopsy Service, which receives biopsies from dentists and ENT surgeons across the province. This population-based biopsy service receives 250 to 300 dysplasia cases annually. Patients with dysplastic lesions were referred to 5 Oral Dysplasia Clinics in Greater Vancouver where they were accrued to the study using written informed consent and a study protocol approved by the Institutional Research Board. Study eligibility required a histologic diagnosis of mild or moderate dysplasia in the oral cavity with no prior history of oral cancer.

The slides of the index biopsy of the 296 patients were reviewed (LZ). The diagnostic criteria were those of WHO and papers from the authorities in the field [1,2]. In a simplistic way as described by Warnakulasuriya et al. [2], when a lesion showed architectural abnormalities and minimal cytological atypia that were limited to the lower third of the epithelium, it was called mild epithelial dysplasia; when a lesion showed architectural abnormalities and minimal to moderate cytological atypia that reached the middle third of the epithelium, it was called moderate epithelial dysplasia. As mentioned before, we had been calling OKND dysplasia, for this study, lesions previously called dysplasia (see second paragraph of introduction) were reclassified as OKND if they showed the following histological features: (1) hyperorthokeratosis; (2) hyperchromatism but no other features of dysplasia; (3) sharp histological margin demarcation from adjacent area by both the hyperorthokeratosis and by the hyperchromatism, and (4) no prominent rete ridges, obvious acanthosis or heavy inflammation (Figure 1). The fourth feature is not a diagnostic feature for dysplasia and was included to rule out reactive lesions, such as those hyperorthokeratotic lesions in the alveolar ridges. Of the 296 lesions, 82 were incisional biopsies; since it was not possible to judge whether there was a sharp margin demarcation, they were excluded, leaving 214 cases for this study.

Demographic, risk habit, and clinical characteristics were collected at study entry. Patients were followed at 6-month intervals and changes to the lesion or risk habits were documented. For this study, the primary endpoint was time from index biopsy 
to histologically confirmed progression to severe dysplasia or higher for progressing cases (occurring at the same anatomical site as the index biopsy) or last follow up date for non-progressing cases. Inclusion of severe dysplasia as the progression endpoint was based on our findings that without treatment, progression to carcinoma in situ and SCC occurred in $59.8 \%$ of patients [13].

The 214 patients had a median follow up time of 44 months. During the study period, 25 (12\%) progressed: 10 to severe dysplasia and 15 to SCC (in situ and invasive).

\section{TB staining}

TB staining consists of three steps: First, the lesion was swabbed with a cotton tip applicator soaked in $1 \%$ acetic acid to remove debris and excess mucosa; then swabbed with an applicator soaked in $1 \% \mathrm{~TB}$ and left for 45 seconds; finally, another swab with $1 \%$ acetic acid, followed by a rinse with water. A stained lesion (dark blue color) was called positive; weak or uncertain uptake of the stain were called equivocal; no stain was called negative (Figure 2).

\section{Assessment of nuclear phenotype score (NPS)}

Serial sections, 4- $\mu \mathrm{m}$ in thickness, were cut from each sample and placed on two glass slides; one was stained with H\&E and the other with Feulgen-Thionin. The area on the H\&E slide selected by the pathologist (L.Z.) for conventional histopathologic diagnosis was the same area circled on the Feulgen-stained slide to be examined with the quantitative tissue phenotype (QTP) imaging system. NPS was determined as described previously [7].

\section{Assessment of molecular risk pattern}

Areas of dysplasia were microdissected for microsatellite analysis. The same protocol for analyzing the LOH markers in chromosome regions 3p14.2; 4q26, 4q31.1; 8p21.3, 8p23.3; 9p21; 11q13.3, 11q22.3; 13q12.3-13, 13q14.3; 17p11.2, and 17p13.1 was applied in this prospective study as described previously [12].

\section{Statistical analysis}

Associations between categorical data were examined with the Chi-Squared tests while unpaired t-tests were used for continuous data. Time-to-progression curves were estimated by the Kaplan-Meier method, and comparisons were performed using a logrank test. Hazard ratios (HRs) and the corresponding 95\% confidence intervals (95\% CI) were determined using Cox regression analysis. $P \leq 0.05$ was considered significant.

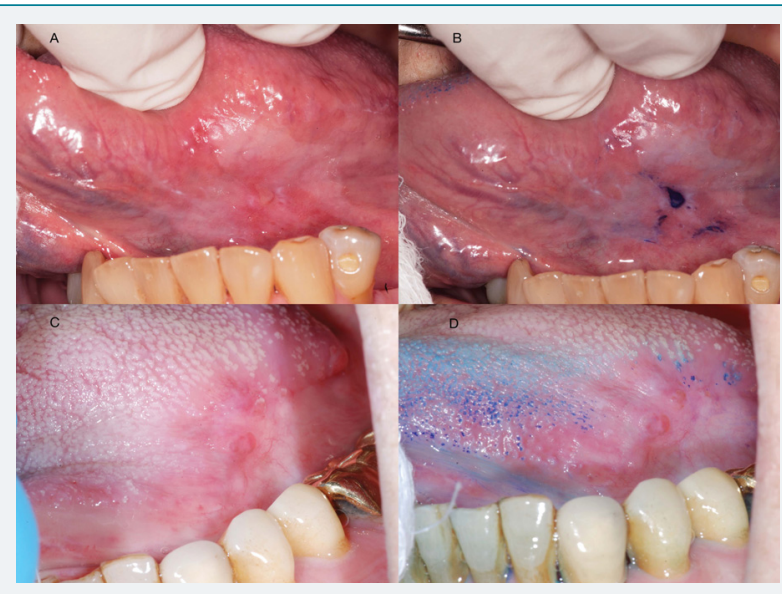

Figure 2: Examples of toluidine blue (TB) staining: Lesion 1 without $(A)$ or with TB staining (B). TB staining result is positive; Lesion 2 without (C) or with TB staining (D). TB staining result is negative. Retention of the TB staining on tongue papillae is a common artifact. 


\section{Results}

Demographic and habit information are shown in table 1. The diagnosis of mild/ moderate dysplasia or OKND was not associated with age at diagnosis, gender, ethnicity or risk habits.

We next compared the clinical lesion characteristics between the two groups (Table 2). Many of the index lesions were biopsied by dentists before the patients were enrolled in our longitudinal study since histological diagnosis of oral dysplasia

\begin{tabular}{|c|c|c|c|c|}
\hline & Total & Mild/Moderate dysplasia & OKND & $\mathbf{P}$ \\
\hline & $N=214$ & $N=194$ & $\mathbf{N}=\mathbf{2 0}$ & value \\
\hline Median age (years) & 57 & 57 & 58 & 0.678 \\
\hline Gender (\% female) & 101 & $93(48 \%)$ & $8(40 \%)$ & 0.639 \\
\hline \multicolumn{5}{|c|}{ Ethnicity } \\
\hline Caucasian & 117 & $159(82 \%)$ & $18(90 \%)$ & \multirow{2}{*}{0.538} \\
\hline Non-Caucasian & 37 & $35(18 \%)$ & $2(10 \%)$ & \\
\hline \multicolumn{5}{|c|}{ Tobacco* } \\
\hline Non-Smoker & 65 & $59(30 \%)$ & $6(30 \%)$ & \multirow{2}{*}{1} \\
\hline Ever Smoker & 149 & $135(70 \%)$ & $14(70 \%)$ & \\
\hline \multicolumn{5}{|c|}{ Alcoholt } \\
\hline Non/light & 159 & $144(79 \%)$ & $15(83 \%)$ & \multirow{2}{*}{0.77} \\
\hline Heavy & 42 & $39(21 \%)$ & $3(17 \%)$ & \\
\hline \multicolumn{5}{|c|}{ Duration of Follow up (month) } \\
\hline Median & 44 & 43 & 49 & \multirow{2}{*}{0.876} \\
\hline Mean & $45 \pm 26$ & $45 \pm 27$ & $42 \pm 21$ & \\
\hline
\end{tabular}

for men. 1 drink $=80 z$ beer $=40 z$ wine $=10 z$ spirits.

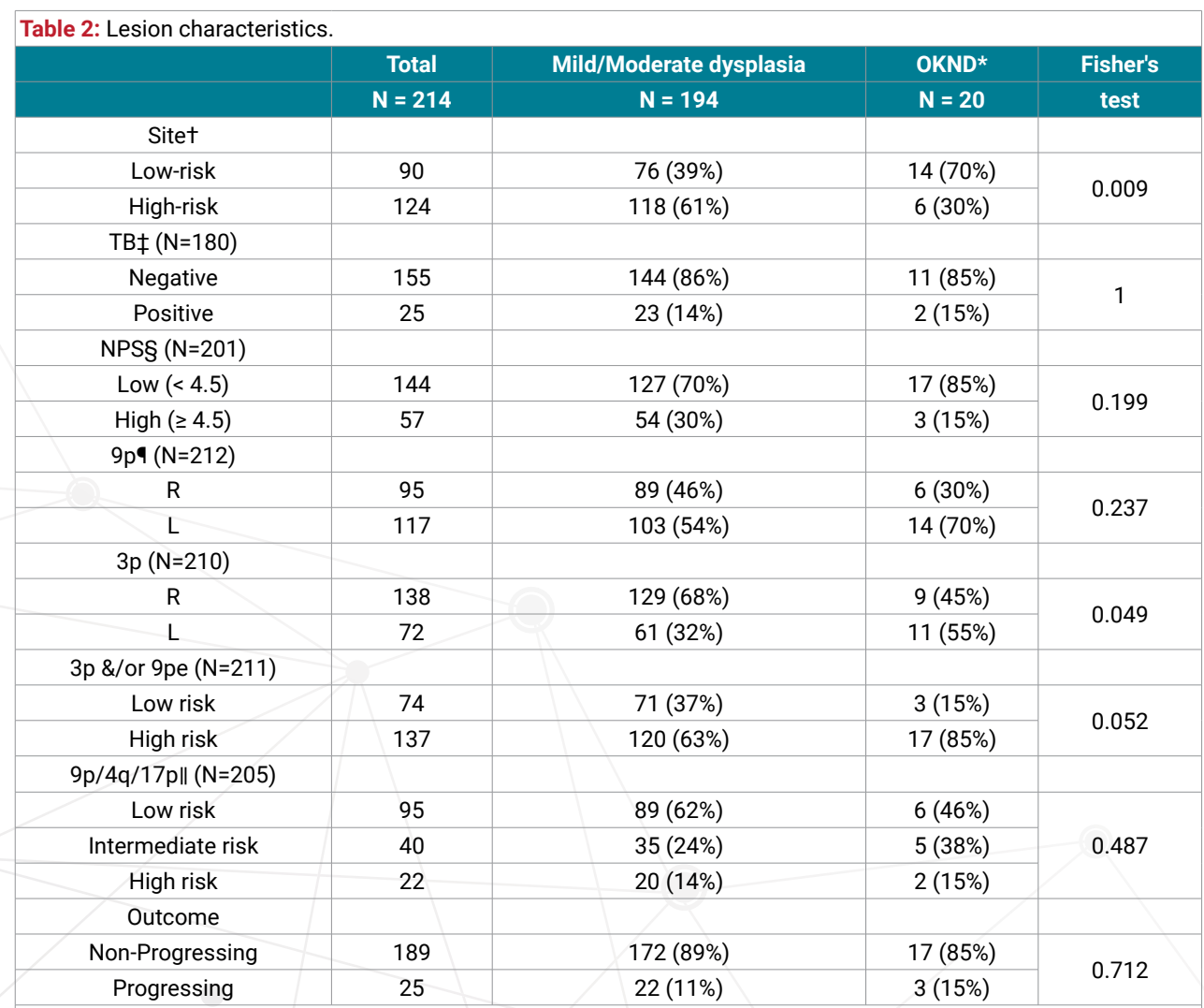

*OKND: orthokeratotic lesion with no dysplasia; +Ventrolateral tongue and floor of mouth are defined as high risk sites, the rest of the oral cavity defined as low risk; ‡TB information is only available for 180 patients and NPS only for 201 patients; $\S$ Total numbers of cases for each reported LOH pattern may not add up to the total number of subjects because of noninformative cases; 9 Risk of progression for specific patterns has been defined previously [12]. Low risk = retained $3 p$ and $9 p$; high risk $=\mathrm{LOH}$ at either $3 p, 9 p$ or both; $\|$ Risk of progression for specific patterns has been defined previously [9]: Low risk $=9 p$ retention; intermediate risk: $9 p \mathrm{LOH}$ with retention at either $4 q$ or $17 p$; high-risk: $9 p \mathrm{LOH}$ with LOH at $4 q$ and $17 p$ 
or OKND was one enrollment requirement. Consequently we did not have clinical pictures for all the index lesions. However, many of OKND were noted to have sharp demarcation for at least for part of the clinical margins, a feature that was also observed for some dysplastic lesions. Due to the lack of a complete set of pictures, this study did not investigate whether histological sharp demarcation of OKNDs also manifested as a clinical sharp demarcation.

A significantly higher proportion of lesions from the dysplasia group were located at a high-risk site (ventrolateral tongue and floor of mouth) as compared to lesions from the OKND group. When the sites were looked at in more detail, a significantly higher percentage of OKND lesions were found to be located on the gingiva, vestibular and cheek regions $(65 \%$ in OKND vs $28 \%$ in mild/moderate dysplasia, $P=0.002$ ), common sites for verrucous lesions (verrucous hyperplasia or carcinoma). We questioned whether some of these OKND were verrucous lesions in a flat leukoplakia stage. We looked at the relationship of dysplasia and OKND with verrucous lesions (whether later developed into a verrucous lesions or have verrucous lesions in other oral sites). A significantly higher proportion of OKND was associated with verrucous lesions as compared to dysplasia ( $50 \%$ OKND vs $8 \%$ dysplasia, $P<0.0001$ ), suggesting a relationship between OKND and verrucous lesions.

TB status was similar between the two groups. Histologically, the proportion of mild/moderate dysplasia with a high NPS score was twice that of the OKND group ( $30 \%$ vs $15 \%)$, although the difference was not significant.

We next compared the two groups for molecular risk patterns (Table 2). For LOH, we looked at two molecular risk models. Using the first model, a higher percentage of loss was noted for the OKND group as compared to the mild/moderate dysplasia group at $9 p, 3 p$ and $3 p \& / o r 9 p$. The difference at $3 p$ was significant $(P=0.049)$ and at $3 p \& /$ or $9 \mathrm{p}$ almost significant $(P=0.052)$. Using the second model, again a higher percentage of intermediate- and high-risk lesions were noted in OKND group as compared to the dysplasia group, although the difference was not significant.

Finally, we compared the probability of progression in the two groups of lesions. No significant difference in outcome was noted between the two groups (Table 2). Kaplan-Meier plots of progression by histological diagnosis showed very similar plots for the two groups of lesions (Figure 3).

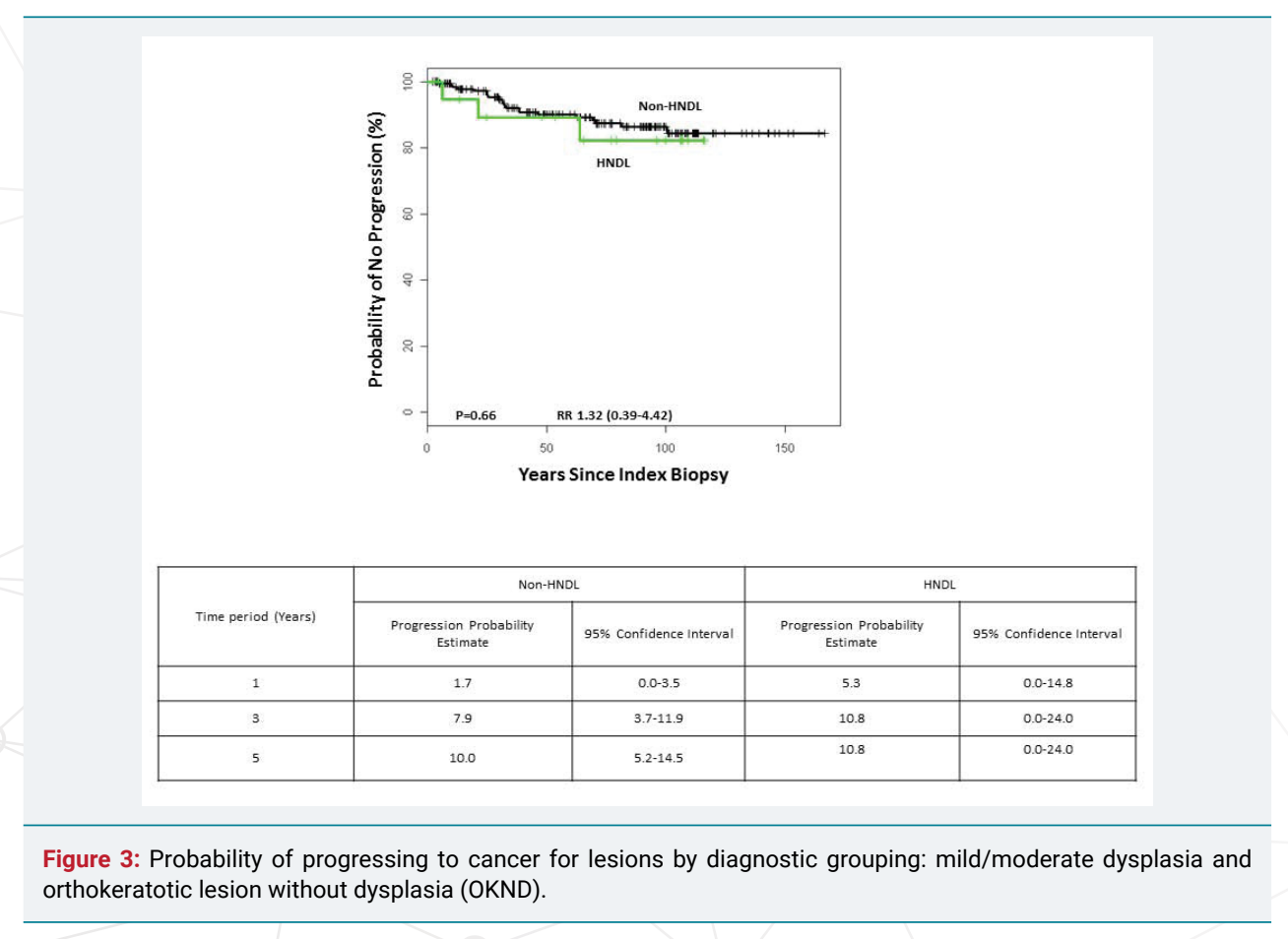




\section{Discussion}

Oral white lesions are common. Histologically these lesions show hyperkeratosis (hyperparakeratosis or hyperorthokeratosis or both) and/or epithelial acanthosis with or without dysplasia. The current gold standard for judging malignant potential of these lesions is by histological assessment of the presence and degree of dysplasia. Unfortunately some of the non-dysplastic lesions are premalignant, particularly those with hyperorthokeratosis.

Supporting evidence for the premalignant potential of hyperorthokeratotic oral lesions has come mainly from clinicians in the form of anecdotal experiences or case studies. The term sublingual keratosis [4], was proposed decades ago to designate such lesions on the floor of mouth and ventrolateral tongue. A subgroup of KUS, a term suggested by Woo et al. [3], designated similar lesions but not limited to the sublingual or floor of mouth. Unfortunately these terminologies are not very helpful in guiding clinical management of the lesions since most clinicians are looking for the word dysplasia to indicate the premalignant nature of these lesions and lack of such word implies no risk.

The premalignant nature of orthokeratotic lesions have been recognized by others. The term 'orthokeratotic dysplasia (OKD)' was coined in a study published by Kobayashi et al. [14], to describe hyperorthokeratotic lesions with granular cell layer (no mentioning whether they were dysplastic). The study investigated 200 cases of oral SCC and 31\% (62/200) of these showed OKD foci. The majority (80\%) were adjacent to the cancer and some (20\%) were separate. Three photomicrographs of OKD were shown: two were dysplastic, one was not. In a follow-up study, Aida et al. [15], used the term to describe hyperorthokeratotic lesions without dysplasia, even though mild atypia was mentioned. Consequently these lesions are similar to the OKND cases in this study. They investigated 20 cases of OKD and confirmed their premalignant nature by finding them frequently associated with oral SCC $(9 / 20,45 \%)$, chromosomal instability and shortened telomeres.

As shown in table 3, the differential diagnosis of OKND lesions from other reactive lesions is not difficult. Reactive keratotic lesions are mostly parakeratotic with some exceptions such as those on the alveolar ridges and rarely other sites. In addition, reactive lesions are very frequently accompanied by thickening of the prickle cell layers, either in the form of acanthosis \&/or proliferation of long rete ridges, frequently square or pointed in shape. Reactive lesions are also more likely to be inflamed whereas OKND lesions generally show absence of heavy inflammation.

\begin{tabular}{|c|c|c|c|c|}
\hline & OKND* & $\begin{array}{l}\text { Benign alveolar } \\
\text { ridge keratosis }\end{array}$ & $\begin{array}{c}\text { Morsicatio mucous } \\
\text { oris }\end{array}$ & Other reactive lesions \\
\hline Keratin & Orthokeratotic & Orthokeratotic & Parakeratotic & $\begin{array}{c}\text { Mostly parakeratotic, } \\
\text { occasionally } \\
\text { orthokeratotic }\end{array}$ \\
\hline $\begin{array}{c}\text { Hyperchromatism } \\
\text { compared to adjacent } \\
\text { epithelium }\end{array}$ & Yes & Probably no & No & No \\
\hline $\begin{array}{l}\text { Sharp demarcation from } \\
\text { adjacent epithelium \& } \\
\text { keratin }\end{array}$ & Yes & Probably no & No & No \\
\hline Epithelial thickness & $\begin{array}{l}\text { No acanthosis \& } \\
\text { sometime atrophic }\end{array}$ & Markedly acanthotic & Markedly acanthotic & $\begin{array}{l}\text { Varying degree of } \\
\text { acanthosis }\end{array}$ \\
\hline Rete ridges & $\begin{array}{l}\text { Absent to slight (not } \\
\text { very long, broad or } \\
\text { pointed) }\end{array}$ & $\begin{array}{l}\text { Marked, could be } \\
\text { broad, square \& } \\
\text { pointed }\end{array}$ & $\begin{array}{l}\text { Marked, could be } \\
\text { broad, square \& } \\
\text { pointed }\end{array}$ & Varying degree \\
\hline $\begin{array}{c}\text { Inflammation in lamina } \\
\text { propria }\end{array}$ & Absent to minimal & Absent to minimal & Absent to minimal & Varying degree \\
\hline
\end{tabular}


In this study we propose that a clonal pattern of changes should be included as a diagnostic criterion for dysplasia. Clonal expansion is one of the fundamental characteristics of the neoplastic process. Some dysplasia, verrucous hyperplasia and SCC show such clonal changes, i.e., sharp demarcation from adjacent normal mucosa. Therefore the inclusion of clonal change as a diagnostic criterion for dysplasia makes sense.

This study compared OKND with mild/moderate dysplasia using multiple cancer risk markers (clinical, TB; histological, NPS; molecular, LOH) and follow-up data.

Clinically, we found no obvious differences between the two groups in TB status. Surprisingly, there were differences in the location of the two groups of lesions. A significantly lower proportion of OKND were noted in the high-risk site when compared with mild/moderate dysplasia ( $30 \%$ vs $61 \% ; P=0.009$ ). Since sublingual keratosis was part of the OKND, we had assumed that a higher percentage of OKND would be located in the high-risk sites. In contrast, a higher percentage of OKND was located on gingiva, vestibular and cheek regions - common sites for verrucous lesions. We also found that OKND are more likely to have either later become verrucous lesions or have verrucous lesions in other oral sites. These findings suggest that at least some of OKND may be precursors of verrucous lesions. The fact that OKND showed similar cancer risk despite location at the low-risk sites supports the premalignant nature of these lesions.

Not surprisingly the mild/moderate dysplasia group showed twice the number of cases with high NPS score as compared to the OKND group, considering the fact that OKND lacks nuclear features of dysplasia. Most important of all, the progression rates between OKND and mild/moderate dysplasia were similar, indicating that OKND lesions have similar malignant potential as mild/moderate dysplasia. We appeal to the research community to study such lesions and to compare the malignant potential of such lesions with dysplastic lesions, particularly in multicenter studies. If further studies confirm our results, we should consider inclusion of sharp demarcation of clonal change as one of the dysplasia diagnostic criterion. Such inclusion would designate lesions currently not called dysplastic as dysplastic, which could provide better guidance to clinicians to improve clinical management of these lesions.

\section{Acknowledgement}

The authors want to thank Dr. Huijun Jiang for her assistance in data collection and statistical analysis. This work was supported by grants R01 DE13124 and R01 DE17013 from the National Institute of Dental and Craniofacial Research.

\section{Author contribution}

L. Zhang, Contributed to conception, design and data analysis, drafted the manuscript; T. Lubpairee, D.-M. Laronde, M. Guillaud, C.-E. MacAulay, and M.-P. Rosin contributed to conception, design, and data analysis and critically revised the manuscript. All authors gave final approval and agree to be accountable for all aspects of the work.

\section{References}

1. Barnes L, Eveson JW, Reichart P, Sidransky D. World Health Organization Classification of Tumours, Pathology \& Genetics of Head and Neck Tumours. Lyon, IARC Press, 2005.

2. Warnakulasuriya S, Reibel J, Bouquot J, Dabelsteen E. Oral epithelial dysplasia classification systems: Predictive value, utility, weaknesses and scope for improvement. J Oral Pathol Med. 2008; 37: 127-133. Ref.: https://tinyurl.com/y7wlgsle

3. Woo SB, Grammer RL, Lerman MA. Keratosis of unknown significance and leukoplakia: a preliminary study. Oral Surg Oral Med Oral Pathol Oral Radiol. 2014; 118: 713-724. Ref.: https://tinyurl.com/y7clxpgq

4. Kramer IR, El-Labban N, Lee KW. The clinical features and risk of malignant transformation in sublingual keratosis. Br Dent J. 1978; 144: 171-180. Ref.: https://tinyurl.com/y74lhmbp

5. Zhang L, Williams M, Poh CF, Laronde D, Epstein JB, et al. Toluidine blue staining identifies highrisk primary oral premalignant lesions with poor outcome. Cancer Res. 2005; 65: 8017-8021. Ref.: https://tinyurl.com/y9hh4kpf 
6. Poh CF, Ng SP, Williams PM, Zhang L, Laronde DM, et al. Direct fluorescence visualization of clinically occult high-risk oral premalignant disease using a simple hand-held device. Head Neck. 2007; 29: 71-76. Ref.: https://tinyurl.com/y93npsns

7. Guillaud M, Zhang L, Poh C, Rosin MP, MacAulay C. Potential use of quantitative tissue phenotype to predict malignant risk for oral premalignant lesions. Cancer Res. 2008; 68: 3099-3107. Ref.: https://tinyurl.com/y9qyhj7s

8. Poh $\mathrm{CF}$, Zhu $\mathrm{Y}$, Chen $\mathrm{E}$, Berean $\mathrm{KW}, \mathrm{Wu} \mathrm{L}$, et al. Unique FISH Patterns Associated with Cancer Progression of Oral Dysplasia. J Dent Res. 2012; 91: 52-57. Ref.: https://tinyurl.com/yd4hysrh

9. Zhang L, Poh CF, Williams M, Laronde DM, Berean K, et al. Loss of heterozygosity (LOH) profilesvalidated risk predictors for progression to oral cancer. Cancer Prev Res. 2012; 5: 1081-1089. Ref.: https://tinyurl.com/y9u7g2ya

10. Garnis C, Rosin MP, Zhang L, Lam WL. Alteration of AKAP220, an upstream component of the Rb pathway, in oral carcinogenesis. Int J Cancer. 2005; 116: 813-819. Ref.: https://tinyurl.com/yay2c495

11. Tsui IFL, Poh CF, Garnis C, Rosin MP, Zhang L, et al. Multiple pathways in the FGF signaling network are frequently deregulated by gene amplification in oral dysplasias. Int J Cancer. 2009; 125: 22192228. Ref.: https://tinyurl.com/y8xptat6

12. Rosin MP, Cheng $X$, Poh $C$, Lam WL, Huang $Y$, et al. Use of allelic loss to predict malignant risk for lowgrade oral epithelial dysplasia. Clin Cancer Res. 2000; 6: 357-362. Ref.: https://tinyurl.com/y7c8t8fs

13. Zhang L, Lubpairee T, Laronde DM, Rosin MP. Should severe epithelial dysplasia be treated? Oral Oncol. 2016; 125-129. Ref.: https://goo.gl/72tPq6

14. Kobayashi T, Maruyama S, Abé T, Cheng J, Takagi R, et al. Keratin 10-positive orthokeratotic dysplasia: A new leucoplakia-type precancerous entity of the oral mucosa. Histopathology. 2012; 61: 910-920. Ref.: https://tinyurl.com/yb4bb2me

15. Aida J, Kobayashi T, Saku T, Yamaguchi M, Shimomura N, et al. Short telomeres in an oral precancerous lesion: Q-FISH analysis of leukoplakia. J Oral Pathol Med. 2012; 41: 372-378. Ref.: https://tinyurl.com/y8on84og 